\title{
Photonic crystal embedded waveguide for compact C-band band-pass filter
}

\begin{abstract}
We report the modelling of a band-pass filter at conventional band (c-band) and the effect of different shapes of photonic crystal $(\mathrm{PhC})$ holes embedded on silicon-on-insulator (SOI) waveguide. The designed embedded waveguides was simulated with LUMERICAL finite different time domain (FDTD) and a filter response with a bandwidth of approximately 30 nm complying with international telecommunication unit (ITU-T) standard was observed. The simulated bandwidth observed was sufficient for guarding against other band interference in telecommunication applications such as wavelength division multiplexing (WDM). The waveguide was designed with a dimension of $600 \mathrm{~nm}$ width $\times 260 \mathrm{~nm}$ height and embedded with PhC of 4 mirrors and 3 cavities. 2 mirrors at both end of the whole structure were designed with less number of holes for obtaining the band-pass filter profile. With a value of lattice constant a, hole radius $r$ and cavity distance $\mathrm{c}$ of 370,115 and $315 \mathrm{~nm}$ respectively, the simulated device spectrum complimented the erbium doped fiber amplifier (EDFA) spectrum to obtain wavelength profile flatness. The $\mathrm{PhC}$ embedded waveguide was tailored to give a 70 percent value of transmission. A flat profile was observed by reducing the photonic crystal hole radii in the middle mirrors. The wavelength band and the bandwidth of the band can be tuned by manipulating the number of mirrors and cavities in waveguide. A different types of $\mathrm{PhC}$ hole shapes were also studied and compared. The transmission quality and bandpass quality with different types of hole shapes show that the circular $\mathrm{PhC}$ shape are superior in comparison with square hole shapes.
\end{abstract}

Keyword: Photonic crystal; Cavity; mirror; Waveguide; Silicon on insulator; C-band; EDFA; WDM; FDTD 